\title{
The Gravitational Attraction between Hemispherical Masses
}

\author{
Jonathan D. Weiss \\ JSA Photonics LLC, 8019 Edith Blvd NE, Albuquerque, NM, USA \\ Email: jondweiss@aol.com
}

How to cite this paper: Weiss, J.D. (2017) The Gravitational Attraction between $\mathrm{He}$ mispherical Masses. Applied Mathematics, 8, 820-834.

https://doi.org/10.4236/am.2017.86064

Received: May 11, 2017

Accepted: June 18, 2017

Published: June 21, 2017

Copyright $\odot 2017$ by author and Scientific Research Publishing Inc. This work is licensed under the Creative Commons Attribution International License (CC BY 4.0).

http://creativecommons.org/licenses/by/4.0/

\section{(c) (i) Open Access}

\begin{abstract}
This paper is a study of the gravitational attraction between two uniform hemispherical masses aligned such that the pair is cylindrically symmetric. Three variations are considered: flat side to flat side, curved side to curved side, and flat side to curved side. Expressions for the second and third variation are derived from the first, with the use of superposition and the wellknown gravitational behavior of a spherical mass as equivalent to a point mass at its center. The study covers two masses of equal diameter and of different diameters, such that one is four times that of the other. Calculations are done for separations from zero to fifty times the radius of the larger of the two, which is effectively the asymptotic limit. It is demonstrated that at any separation, the force can be expressed as if the two hemispheres were point masses separated by a certain distance. Expressions for that distance and the location of the (fictitious) point masses within each hemisphere are presented. Unlike the case of two spherical masses, the location within their respective hemisphere is not necessarily the same for each point and both are dependent upon the separation between the two hemispheres. The calculation for the first variation is done in two ways. The first is a "brute force" multi-dimensional integral with the help of Wolfram Mathematica. The second is an axial expansion for the potential modified for off-axis locations by Legendre polynomials. With only a few terms in the expansion, the results of the second method are in extremely good agreement with those of the first. Finally, an interesting application to a split earth is presented.
\end{abstract}

\section{Keywords}

Gravitational Attraction, Hemispherical Solids, Legendre Polynomials, Split Earth

\section{Introduction}

With at least one exception, a perusal of online publications of the (Newtonian!) 
gravitational attraction between solid masses yields discussions of a pedagogical nature concerning spherically symmetric solids and shells. In a few cases, the force between a point mass and a disk or a hemisphere is derived for the point mass placed along the center line of the non-spherical object. The exception is a calculation of the force between two hemispheres with their flat surfaces in contact, as in the case of the earth [1]. In addition to considering pairs of nonspherically symmetric masses, this paper generalizes the discussion in [1] to the force between two hemispheres of varying separation, such that the pair forms a cylindrically-symmetric configuration. Furthermore, two additional relative orientations are analyzed, along with the one involving both flat surfaces facing each other, as in [1]. These involve the curved surfaces facing each other, or a curved surface facing a flat surface. As a further generalization, two sets of sizes are also considered. The first set involves both hemispheres of the same diameter, while the second involves diameters that differ from each other by a factor of four. The situation is shown in Figure 1 , where $2 A$ and $2 B$ merge when $B=A$. The symmetry dictates that the net force between the two hemispheres is in the vertical direction. Calculations of the force will be done for separations between the closest two points, $\mathrm{S}$, such that $0 \leq \mathrm{S} \leq 50 \mathrm{~A}$, essentially the asymptotic limit.

Figure 2 illustrates how the force associated with the second two arrangements can be derived from the force associated with the flat-to-flat arrangement through linear superposition of forces. Thus only the first arrangement requires a nontrivial calculation. The arrangements involving a sphere and a hemisphere are equivalent to a point source outside the hemisphere and positioned along its center line. This calculation is comparatively easy to perform.

In addition to the force calculations, it will be shown that the force can be expressed in the form of Newton's law of gravitation for point masses, $G M_{A} M_{B}$ l $\left(R_{e f f}\right)^{2}$, where $R_{\text {eff }}$ is an effective separation between the two point masses, each having the same mass as the associated hemisphere. Only the magnitude of the force is considered here. This situation is shown in Figure 3 for the flat-to-flat configuration. The location of each of the two fictitious point masses is within its respective hemisphere at a position $\alpha_{A} A$ or $\alpha_{B} B$ from the flat surface. The two $\alpha$ 's

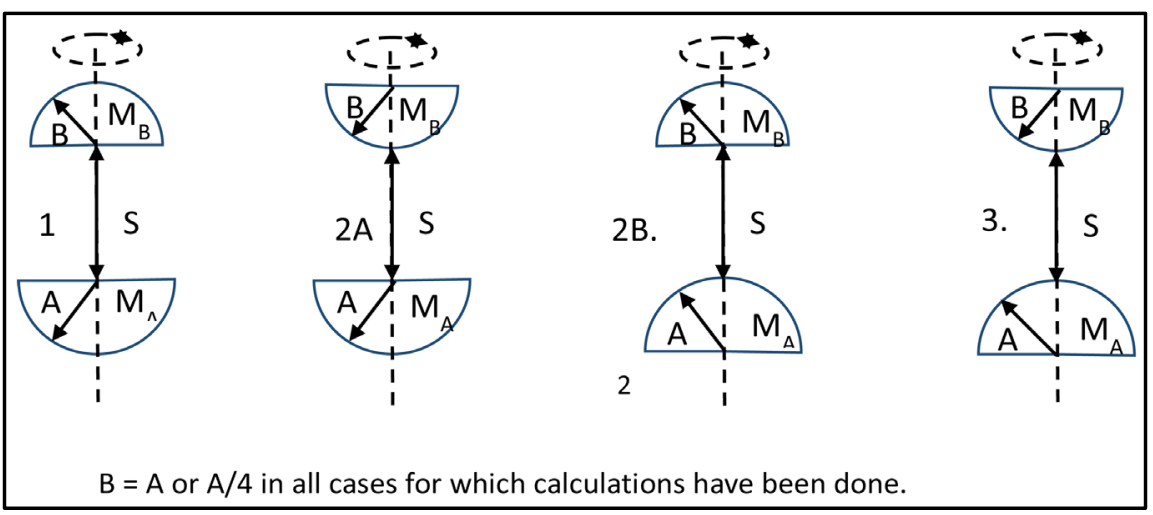

Figure 1. The four configurations analyzed in this paper, showing the axis of symmetry. The mass and radius of the lower (upper) hemisphere are $M_{A}\left(M_{B}\right)$ and $A(B)$, respectively. 

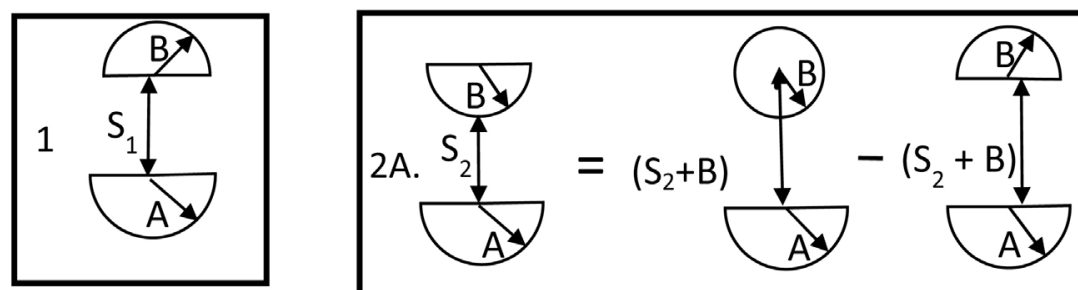

(a)

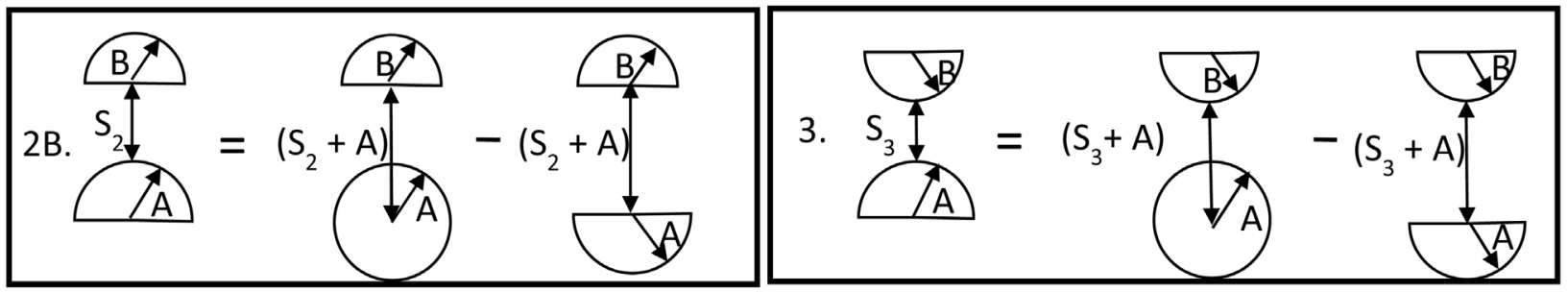

(b)

Figure 2. Illustrations of how configurations $2 A, 2 B$, and 3 can be determined from the first configuration, thereby simplifying the analysis.

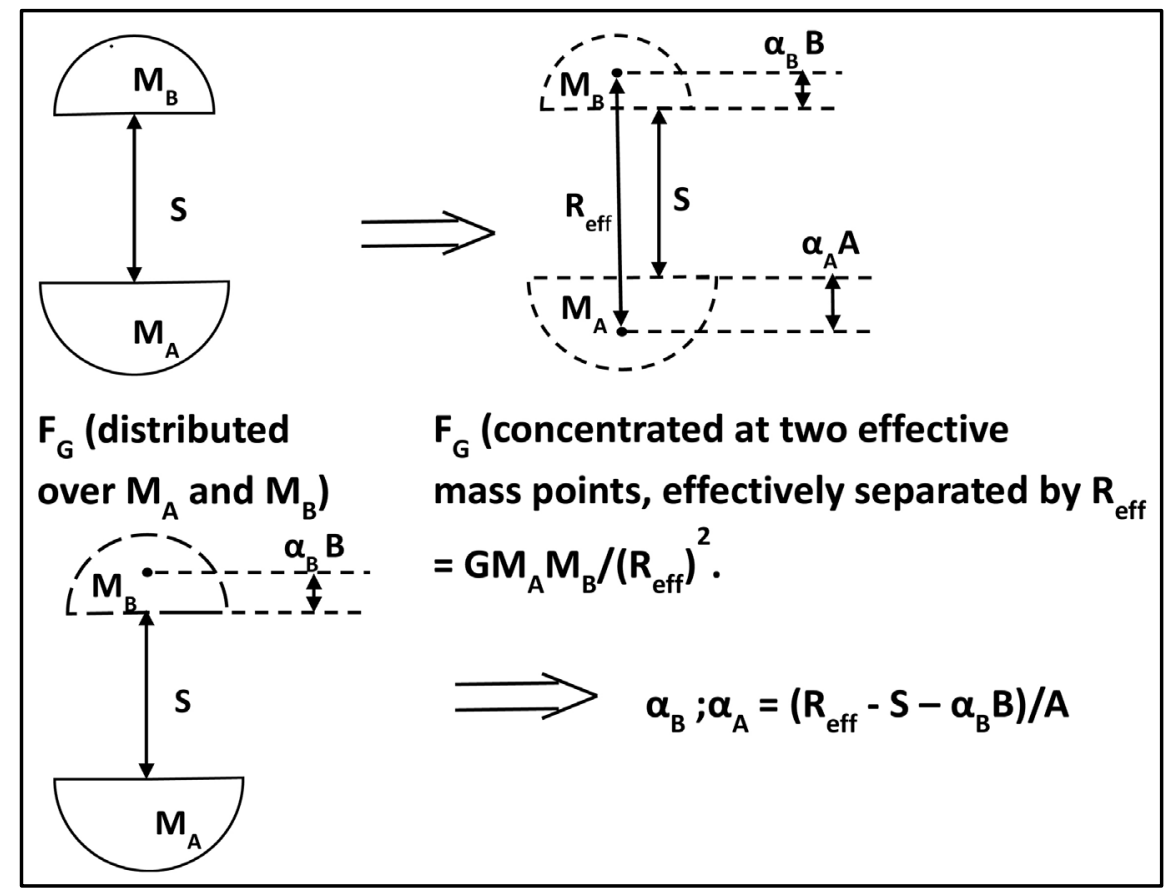

Figure 3. Illustration of how the force can be expressed in terms of an effective separation between two point masses and how to determine the location of those point masses within the hemispheres.

refer to the fraction of the radius, $A$ or $B$, where the point mass is located. If a curved surface faces down, the position will be measured from that curved surface. In all cases, the position will be calculated as a function of $S$ and shown to approach the center of mass of the respective hemisphere at infinite separation. The separation of $50 \mathrm{~A}$ is fully adequate to demonstrate that point. This center-of-mass limit ((3/8) $A$ (or $B$ ), measured from the flat surface, and (5/8) $A$ (or 
$B$ ), measured from the curved surface) can be proved analytically and can be viewed as a test of the accuracy of the calculation. Their position at any separation will be calculated, as shown in the bottom of Figure 3, by replacing one of the hemispheres by its equivalent point mass at the unknown position $\alpha_{B} B$ and calculating the force between it and the other hemisphere. That force between the two will be equated to the force previously calculated between the two hemispheres, thus determining $\alpha_{B} B$. Since $\alpha_{A} A+\alpha_{B} B+S=R_{\text {eff }}$, the other location is determined.

\section{Calculations}

\subsection{Preliminary}

First, let us be specific about the superposition of collinear forces, at least in one case. Others are similar. Assuming the form $G M_{A} M_{B} /\left(R_{\text {eff }}\right)^{2}$ and referring to Figure 2, panel $2 A$, we find that

$$
\frac{1}{\left[R_{\text {eff }}\left(c f ; S_{2}\right)\right]^{2}}=\frac{2}{\left[R_{e f} f\left(p f ; S_{2}\right)\right]^{2}}-\frac{1}{\left[R_{e f f}\left(f f ; S_{2}+B\right)\right]^{2}}
$$

where, in order of their appearance, " $c f$ " refers to curved-flat; " $p f$ " refers to point-flat (because the sphere acts as a point source), and " $f f$ " refers to flat-flat, as also shown in the first panel. The separation for each $R_{e f f} S_{2}$ and $S_{2}+B$, are also shown. The factor of 2 exists because the mass of the sphere is twice that of the hemisphere.

Next, we present the formulas for the magnitude of the force between a sphere and a hemisphere required in Figure 2(A), Figure 2(B), and 3 of Figure 2. In panels $2 A$ and $2 B$, the flat surface of the hemisphere faces the sphere, but with $A$ and $B$ reversed. In panel 3, the curved side of the hemisphere faces the sphere. $A$ simple integration yields the following for the force, $F_{p s}$ in Figure 2(A):

$$
F_{p f}=G M_{A}\left(2 M_{B}\right)\left[\frac{3}{A^{3}}\right]\left[\left(\frac{1}{3 S^{2}}\right)\left\{\left(2 S^{2}-A^{2}\right) \sqrt{A^{2}+S^{2}}+3 A S^{2}+\left(A(A-S)-2 S^{2}\right)(A+S)\right\}\right]
$$

where we identify the multiplier of $\left[G M_{A}\left(2 M_{B}\right)\right]$ with $\left(1 / R_{e f f}\right)^{2}, S=S_{2}+B$, and $M_{A}$ and $M_{B}$ are the two hemispherical masses. In the case of panel $2 \mathrm{~B}, A$ and $B$ are reversed in the above formula. The equation for the force between the curved side facing the sphere and the sphere can be obtained by a similar integration, resulting in a similar formula. More simply, it can be obtained by subtracting from the force between two spheres the force between the sphere and the hemisphere (flat side facing the sphere), as shown in Figure 4 . The force between the two spheres is simply proportional to $4 /\left(S_{3}+A+B\right)^{2}$.

\subsection{Force between Two Hemispheres: Flat Surface Facing Flat Surface}

As was stated earlier, this situation is the only one requiring a nontrivial calculation. It was performed in two ways. The first involves a direct integration of the force between all of the points in one hemisphere and all of the points in the 


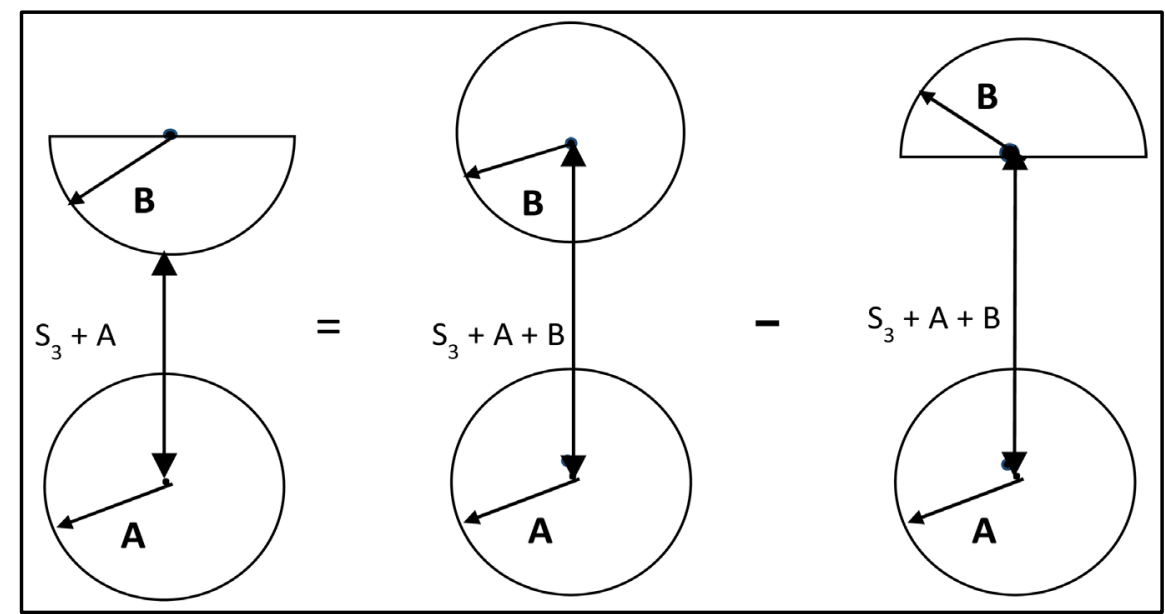

Figure 4. An illustration of the use of superposition to determine the force between a sphere and a hemisphere, curved side facing forward, needed to analyze configuration 3 in Figure 1.

other. Because integration over two volumes was required, it started out as a six-fold integration. However, azimuthal symmetry reduced it by one integral and another integral could be done analytically, thus reducing the problem to a still-unpleasant four-fold integration. The second way involved expansions of the potential created by one hemisphere along its axis for positions $z \leq A$ and $z \geq$ $A$. The off-axis values were then expressed in terms of the original expansion multiplied by Legendre polynomials, as in electrostatics.

\section{Direct Integration Method}

The direct integration method was performed with respect to Figure 5, where two hemispheres, along with the two sets of dimensions and the separation between them are shown.

The basic integral expression for the point-to-point (pp) force, $F_{p p}$, is:

$$
F_{p p}=G \rho_{A} \rho_{B} \iint \frac{\mathrm{d} V_{A} \mathrm{~d} V_{B} \cos \varphi}{R^{2}}
$$

where $V_{A}$ and $V_{B}$ are the volume elements in their respective hemisphere; $R$ is the separation between any two points in different hemispheres; $\varphi$ is the angle between $R$ and the vertical; and $\rho_{A(B)}$ is the mass density of hemisphere $A(B)$, assumed to be different for the sake of generality. By symmetry, the force is in the vertical direction. This equation can be written in an explicit form:

$$
F_{p p}=G \rho_{A} \rho_{B} \int_{0}^{B} \mathrm{~d} z_{B} \int_{0}^{\left(B^{2}-z_{B}^{2}\right)^{0.5}} R_{B} \mathrm{~d} R_{B} \int_{0}^{2 \pi} \mathrm{d} \varphi_{B} \int_{0}^{2 \pi} \mathrm{d} \varphi_{A} \int_{0}^{A} \mathrm{~d} z_{A} \int_{0}^{\left(A^{2}-z_{A}^{2}\right)^{0.5}} R_{A} \mathrm{~d} R_{A} \frac{\left(z_{A}+z_{B}+s\right)}{\left[\left(z_{A}+z_{B}+s\right)^{2}+r_{A}^{2}+r_{A}^{2}-2 R_{A} R_{B} \cos \varphi_{A}\right]^{\frac{3}{2}}}
$$

Writing the equation in the form of the product of the two point masses divided by the square of an effective separation between them scaled by the radius $A\left(1 / R_{\text {eff } A}\right)^{2}$, we obtain the following complicated expression:

$$
\left(\frac{1}{R_{e f f, A}}\right)^{2}=\frac{9}{\pi} \int_{0}^{1} \mathrm{~d} z_{b} \int_{0}^{\left(1-z_{b}^{2}\right)^{0.5}} R_{b} \mathrm{~d} R_{b} \int_{0}^{\pi} \mathrm{d} \varphi_{a} \int_{0}^{1} \mathrm{~d} z_{a}\left(z_{a}+b z_{b}+s_{a}\right)[]
$$




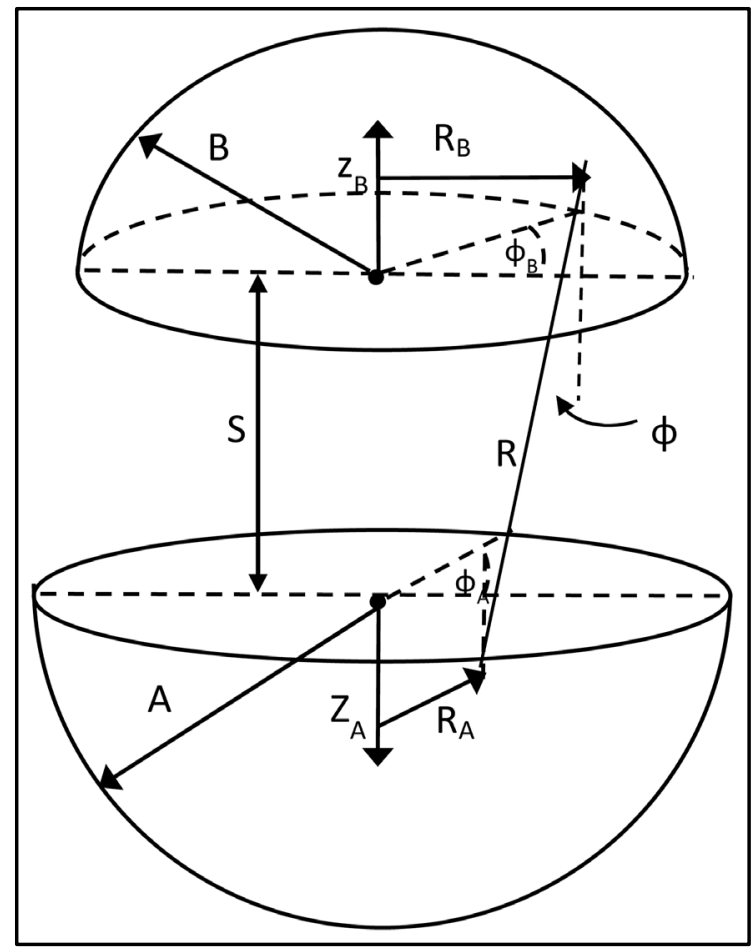

Figure 5. The geometry needed for the direct integration method. The angles $\varphi_{A}$ and $\varphi_{B}$ are azimuthal, defining the direction between the horizontal vectors, $r_{A}$ and $r_{B}$ and the dotted reference lines.

where

$$
\begin{aligned}
{[]=} & \frac{\left[\left(z_{a}+b z_{b}+s_{a}\right)^{2}+\left(b R_{b}\right)^{2}\right]^{1 / 2}}{\left(b R r_{b} \sin \varphi_{a}\right)^{2}+\left(z_{a}+b z_{b}+s_{a}\right)^{2}} \\
& +\frac{\left(b R_{b} \cos \varphi_{a}\right)\left(1-z_{a}^{2}\right)^{1 / 2}-\left(\left(z_{a}+b z_{b}+s_{a}\right)^{2}+\left(b R_{b}\right)^{2}\right)}{\left(\left(b R_{b} \sin \varphi_{a}\right)^{2}+\left(z_{a}+b z_{b}+s_{a}\right)^{2}\right) X^{\frac{1}{2}}}
\end{aligned}
$$

And where

$$
X=\left(1-z^{2}\right)-\left(2 b R_{b} \cos \varphi_{a}\right)\left(1-z_{a}^{2}\right)^{1 / 2}+\left(\left(z_{a}+b z_{b}+s_{a}\right)^{2}+\left(b R_{b}\right)^{2}\right)
$$

$R_{\text {eff }, A}=R_{\text {eff }} / A$. All the other linear parameters in the expression are similarly scaled and use similar notation (e.g., $s_{a} \equiv S / A ; b=B / A ; Z_{a}=Z_{A} / A ; r_{a}=R_{A} / A ; z_{b}=$ $\left.Z_{B} / B ; r_{b}=R_{B} / B\right)$. The integral over the azimuthal angle, $\varphi_{B}$, forthe upper hemisphere was simply replaced by $2 \pi$, and the integral over $r_{A}$ was doable analytically. Thus, as stated above, the 6-fold integral was reduced to a 4 -fold integral. Since the 4-fold integral did not appear to be analytically evaluable, it was numerically evaluated by using the Wolfram Mathematica command "NIntegrate" as applied to multiple integration. The results of the direct integration are shown in Figure 6 for $b=1$ and $b=1 / 4$. Configuration 1 refers to the first panel in Figure 1. The basic behavior is the same for both values of $b$ : a rapid drop off for small values of $S_{A}$, followed by a transition to very small values of $\left(1 / R_{\text {eff }, A}\right)^{2}$ at large values of 


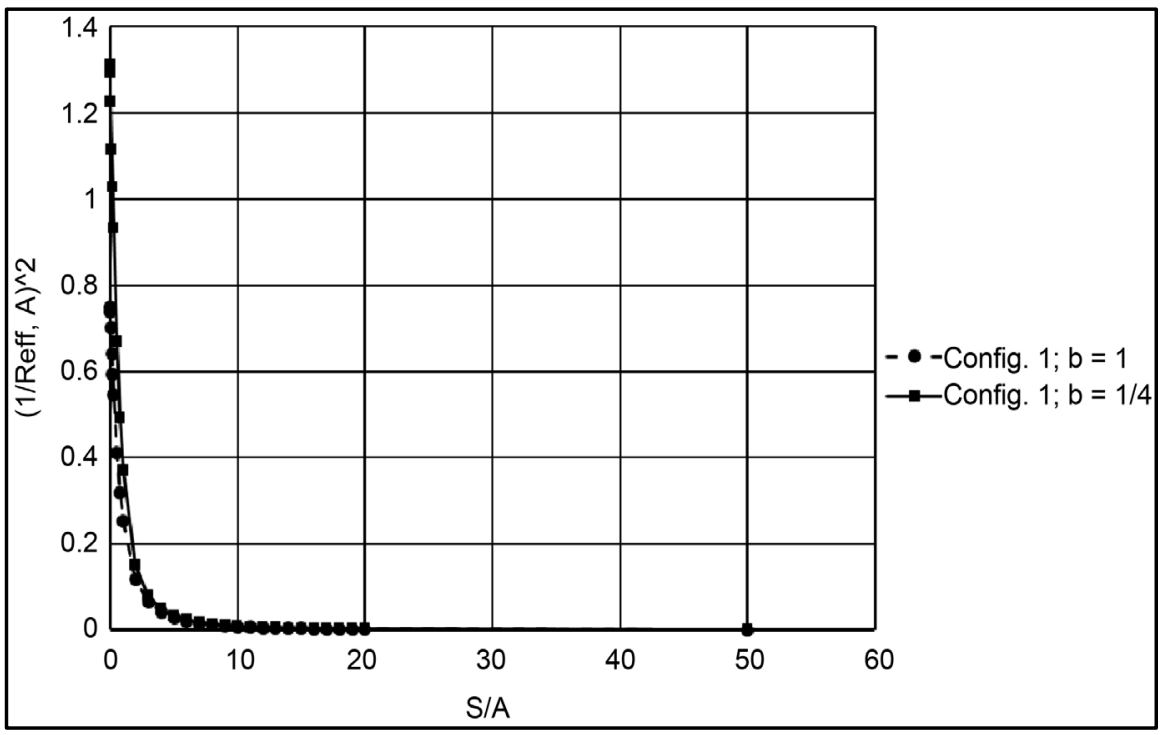

Figure 6. The inverse square of the scaled separation, $\left(1 / R_{\text {eff } A}\right)^{2}$, for the $1: 1$ and the $0.25: 1$ of configuration 1 over separations from 0 to $50 \mathrm{~A}$. The values for $b=1 / 4$ are larger than for $b=1$.

$S_{A}$. The values for $b=1 / 4$ are consistently larger than those for $b=1$. At $S=0$, for example, the two are 1.31 and 0.75 , respectively. The force for $b=1$ is considerably larger, however, because the product of the masses is 64 times as large. Given the value of 0.75 for $\left(1 / R_{\text {eff }, A}\right)^{2}$ with $b=1$, an expression for the magnitude of the force between the two hemispheres in contact with one another is $(1 / 3)$ [ $\left.G\left(\pi \rho A^{2}\right)^{2}\right]$, where $\rho$ is the mass density of both hemispheres. This is the same result obtained in [1] by different methods from the one used here (see Equations (5) and 19 of that reference).

\subsection{Gravitational Force between Two Halves of a Sphere}

Since the gravitational force between the two halves of a solid sphere, such as the earth, is of some interest to inquiring minds [2], we shall consider two applications of the result immediately above. The first concerns how the gravitational force between two contacting spheres, each of the same mass as the individual hemispheres, compares to the force between the two hemispheres. Since the ratio of the radii, sphere to hemisphere, is $1 / 2^{1 / 3}$, the ratio of the two forces, spheres to hemispheres, can easily be deduced as $\left(2^{2 / 3} / 3\right) \approx 0.53$. Thus, the force between the two hemispheres is almost twice that of the two spheres. The second application is concerned with the rotation rate of the earth required to separate its two halves under only the combined action of the fictitious centrifugal force and gravity. The calculation is done with reference to Figure 7 , in which is shown a sphere of radius $A$ split along a plane perpendicular to the plane of the paper and passing through the central axis of rotation. The split sphere is rotating with angular velocity $\omega$. The coordinates $(r, \varphi, z)$ define the position of a rotating point. The total "centrifugal force", $F_{c}$ is obtained by integrating the component of the force perpendicular to the interface plane over the hemisphere. 


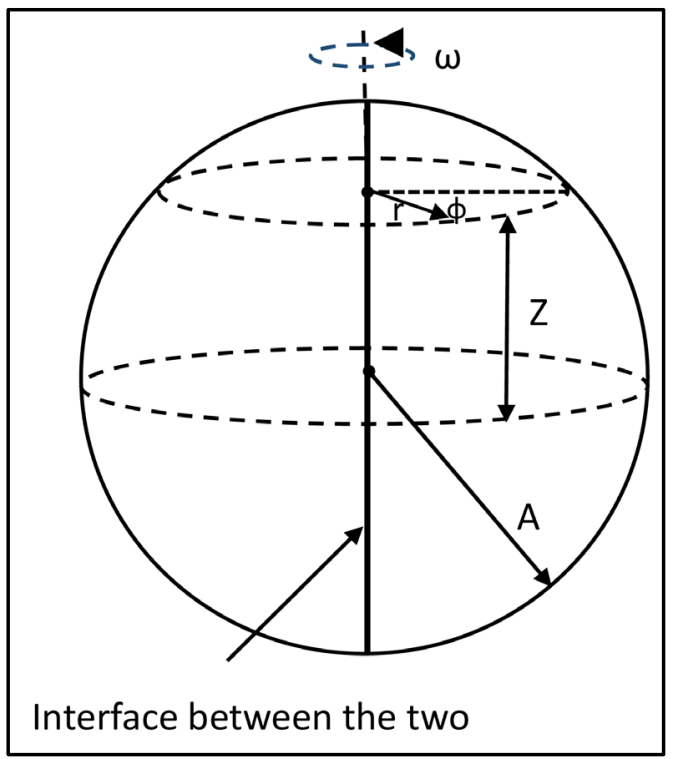

Figure 7. The geometry of gravitational force between the two halves of a solid sphere, such as the earth.

$$
F_{c}=2 \omega^{2} \rho \int_{0}^{A} \mathrm{~d} z \int_{0}^{\left(A^{2}-z^{2}\right)^{1 / 2}} r^{2} \mathrm{~d} r \int_{-\frac{\pi}{2}}^{\frac{\pi}{2}} \cos \varphi \mathrm{d} \varphi=\frac{\pi}{4} \omega^{2} \rho A^{4}
$$

Equating $F_{c}$ to $\left[G\left(\pi \rho A^{2}\right)^{2}\right] / 3$, solving for $\omega$, and expressing $\omega$ as $2 \pi N_{r}$, where $N_{r}$ is the number of rotations per second, we obtain $N_{r}=[G \rho /(3 \pi)]^{1 / 2}$. This results in $\approx 20 \times 10^{-5}$ rotations/sec. required to just separate the two halves of the sphere. The earth's rotational speed $\approx 1.16 \times 10^{-5}$ rotations/sec., which $\approx(1 / 17)$ of the required speed. Of course, the separation speed would be much greater if the cohesion within the earth, which serves a kind of geological corpus callosum, were taken into account. Furthermore, tidal friction is causing the earth's rotational speed to slowly diminish. Thus, we have nothing to worry about.

\subsection{An Array of Results}

In this section, we present a graphical array of results, in addition to the one already presented in Figure 6. The graphical array will be of $\left(1 / R_{e f f, A}\right)^{2}$ vs. $S_{A}$ for the various configurations in Figure 1 and of Alpha (the spelled out version of the Greek letters in Figure 2) for those same configurations. Figure 8 is a more detailed view of the behavior of $\left(1 / R_{e f f, A}\right)^{2}$ for $S_{A} \leq 3, b=1$, and for its three distinguishable configurations. The dotted line is added to show the linear behavior in the immediate vicinity of $S_{A}=0$. The ordering in size is consistent with the amount of mass in one hemisphere that is close to the other hemisphere. That order is flat-to-flat, flat-to-curved, curved-to-curved. Figure 9 is similar to Figure 8, but with $b=0.25$. For these graphs, $\left(1 / R_{e f f, A}\right)^{2}$ is larger than in Figure 8 because one of the hemispheres is so much smaller. The ordering in size in Figure 9, in general, is also consistent with the amount of mass in one hemisphere that is close to the other. Curiously, however, there is a cross over between con- 


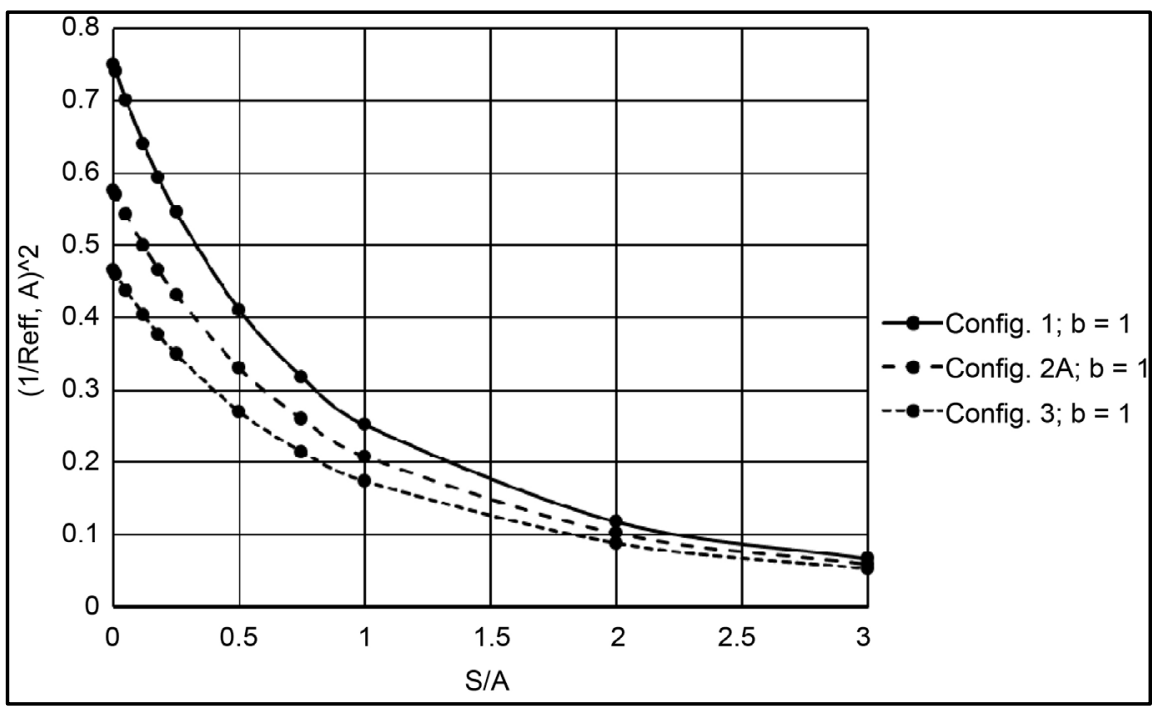

Figure 8. A view of $\left(1 / R_{\text {eff }, A}\right)^{2}$ for the three distinguishable configurations for $b=1$ over the range over separations from 0 to 3 .

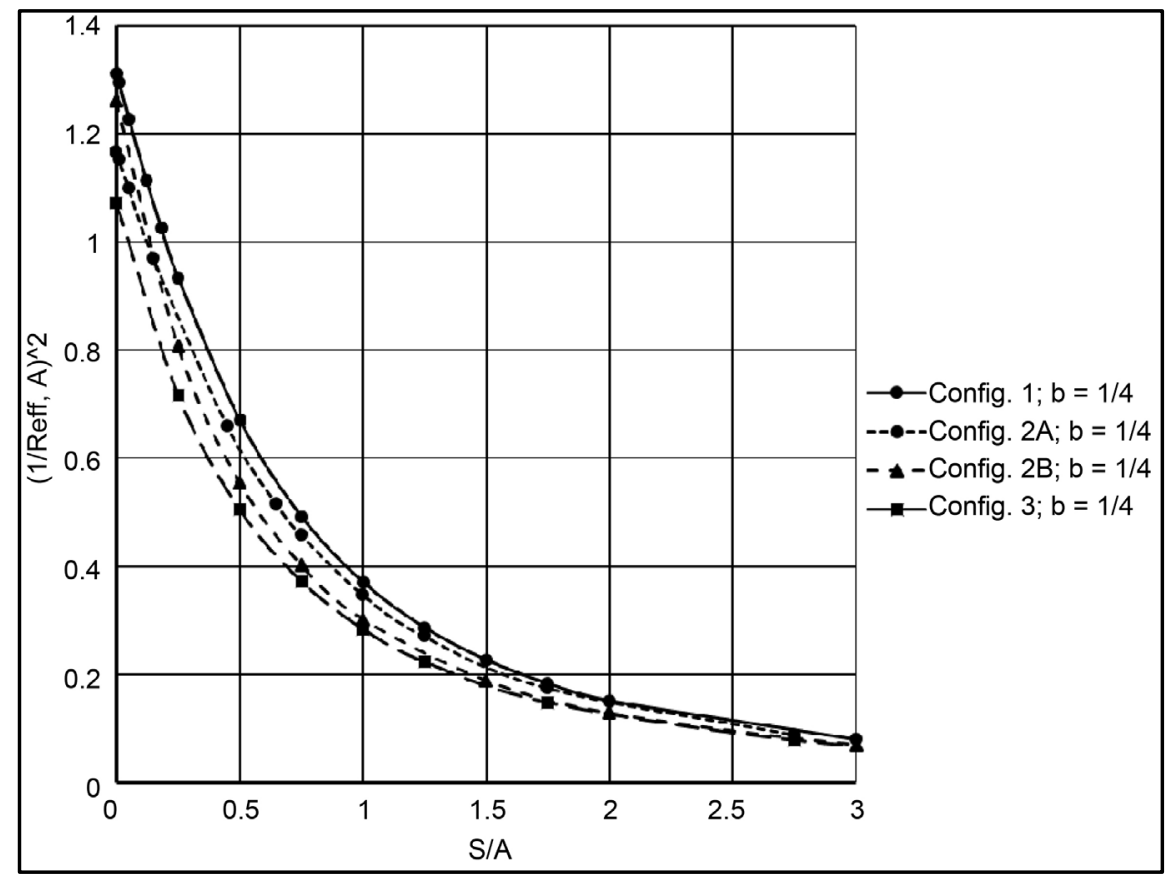

Figure 9. Similar to Figure 8 , but for $b=0.25$. Configurations $2 \mathrm{~A}$ and $2 \mathrm{~B}$ are now distinguishable.

figurations $2 A$ and $2 B$ at $S_{A}=0.25$, which is maintained down to $S_{A}=0$. Figure 10 and Figure 11 are the same as Figure 8 and Figure 9, except that the entire range of values are shown on a log-log scale. The dashed line in Figure 10 shows how the behavior at large values of $S_{A}$ varies as $1 / S_{A}^{2}$. Figure 12 and Figure 13 illustrate the behavior of Alpha for various configurations and the two values of b ( 1 and 1/4). Alpha 1 (or Alpha 1/4) is the value of Alpha for a hemispherical radius $=1$ (or 1/4). As expected from the comment in the introduction, this parameter approaches $(3 / 8)$ or $(5 / 8)$ for large values of $S_{A}$, depending on whether 


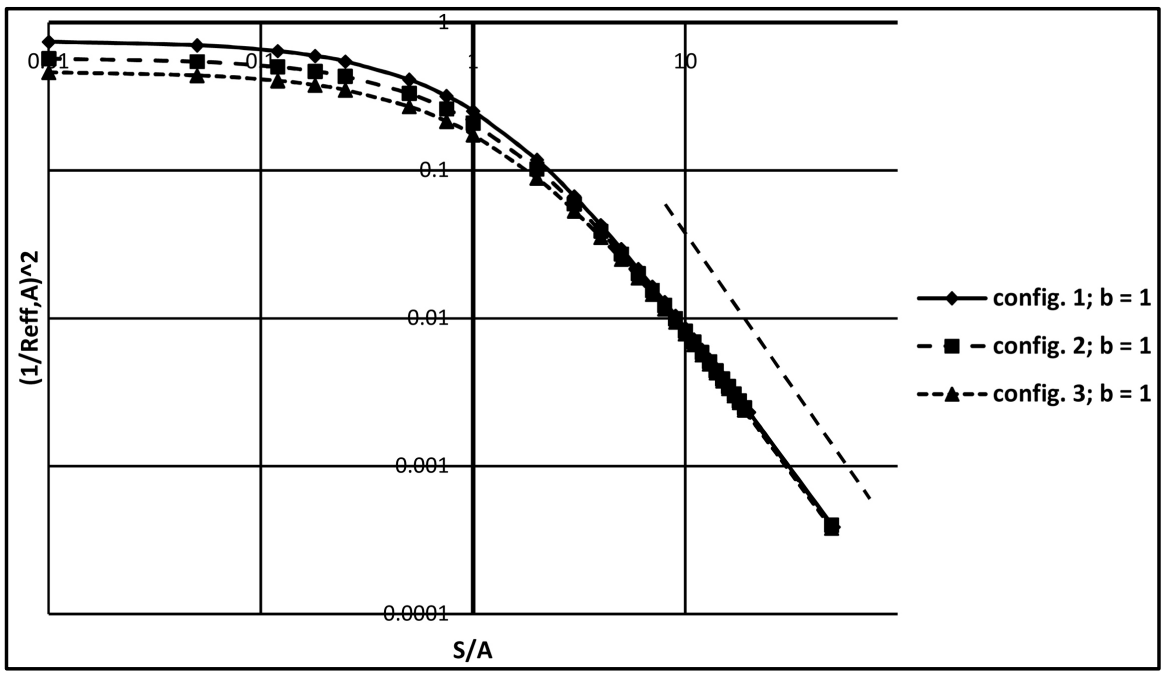

Figure 10. The same as Figure 8, but on a log-log scale for separations between 0 and 50 A. The dashed line behaves as $\left[(1 /(S / A)]^{2}\right.$.

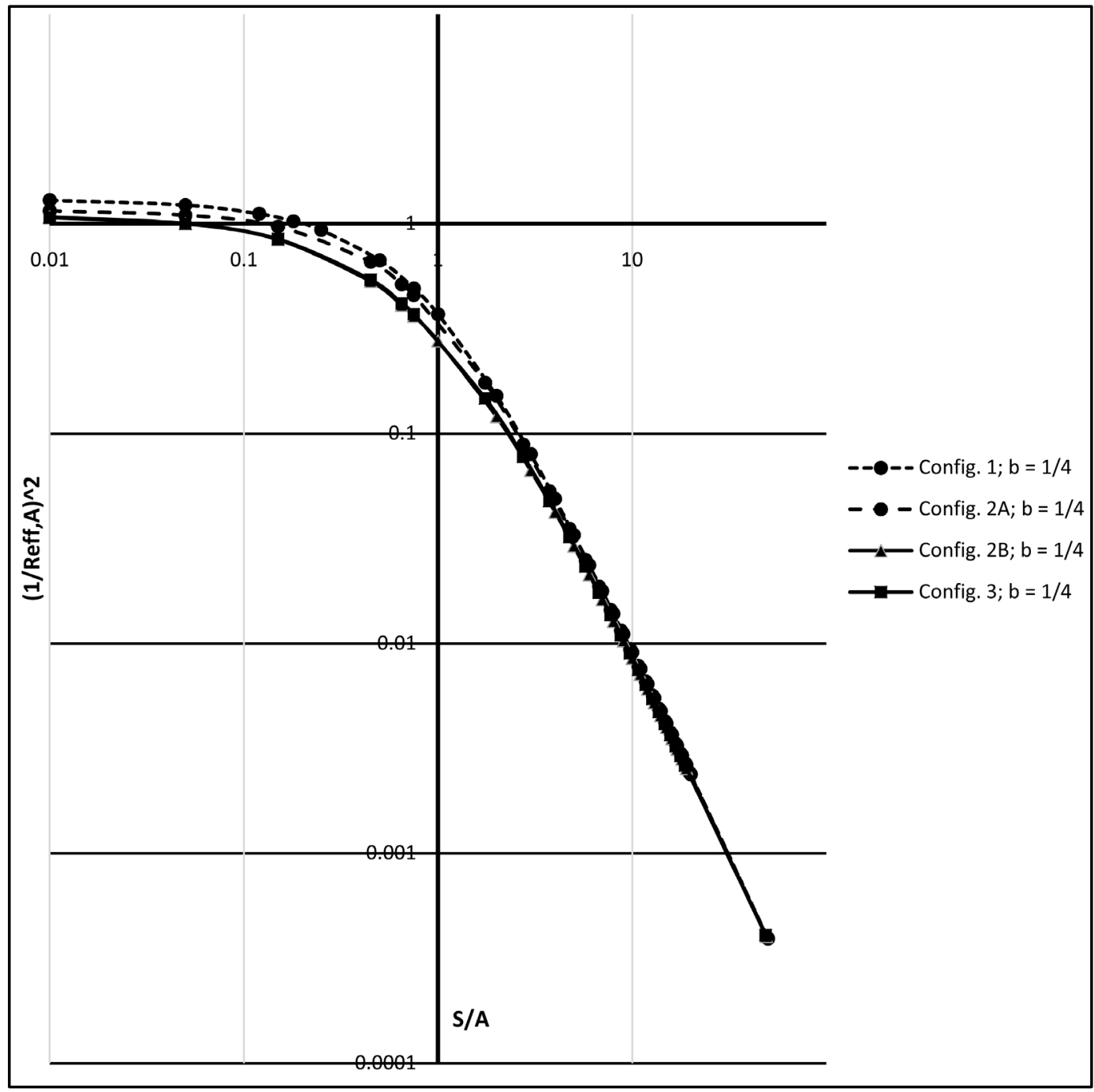

Figure 11. The same as Figure 9, but on a log-log scale for separations between 0 to $50 \mathrm{~A}$. Configurations $2 B$ and 3 are indistinguishable on this log-log graph. 


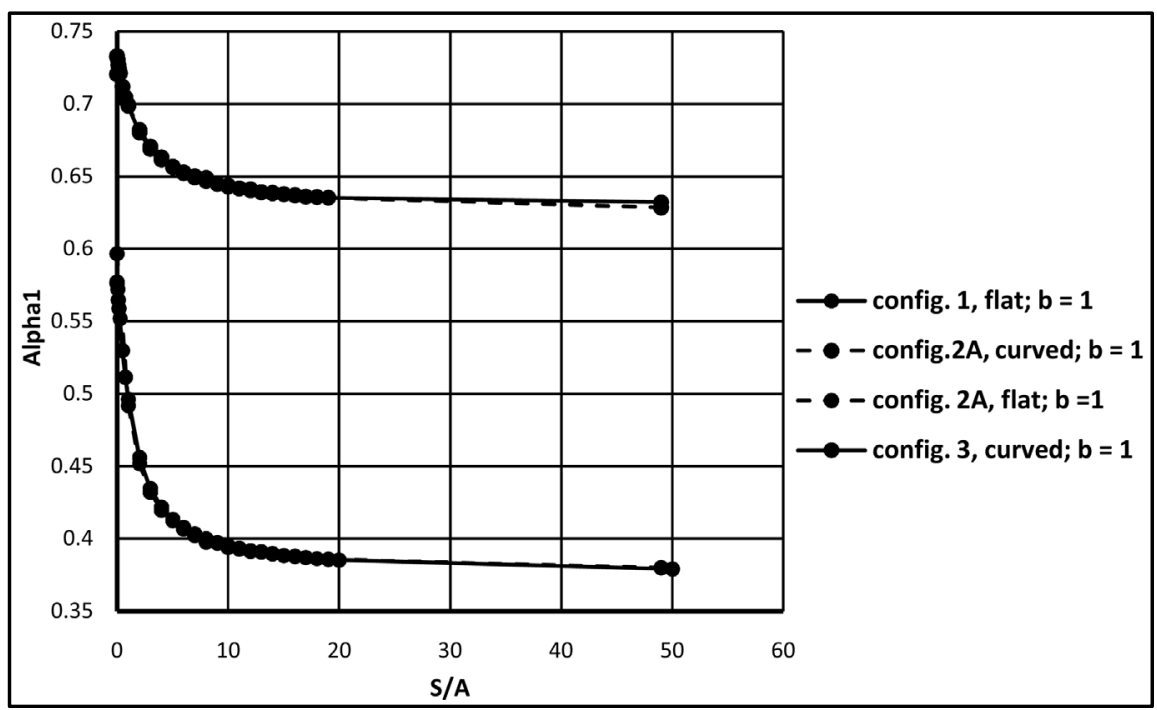

Figure 12. The top two curves, configuration $2 A$ curved and configuration 3 curved, are indistinguishable on this graph. However, Alpha 1 for configuration 3 is slightly greater than that for configuration $2 A$ because the force between the two hemispheres is slightly greater in configuration $2 A$, as is made clear in Figure 1 . A similar comment applies to the other pair of curves, where Alpha 1 for configuration 2 flat is slightly greater than that for configuration 1 flat.

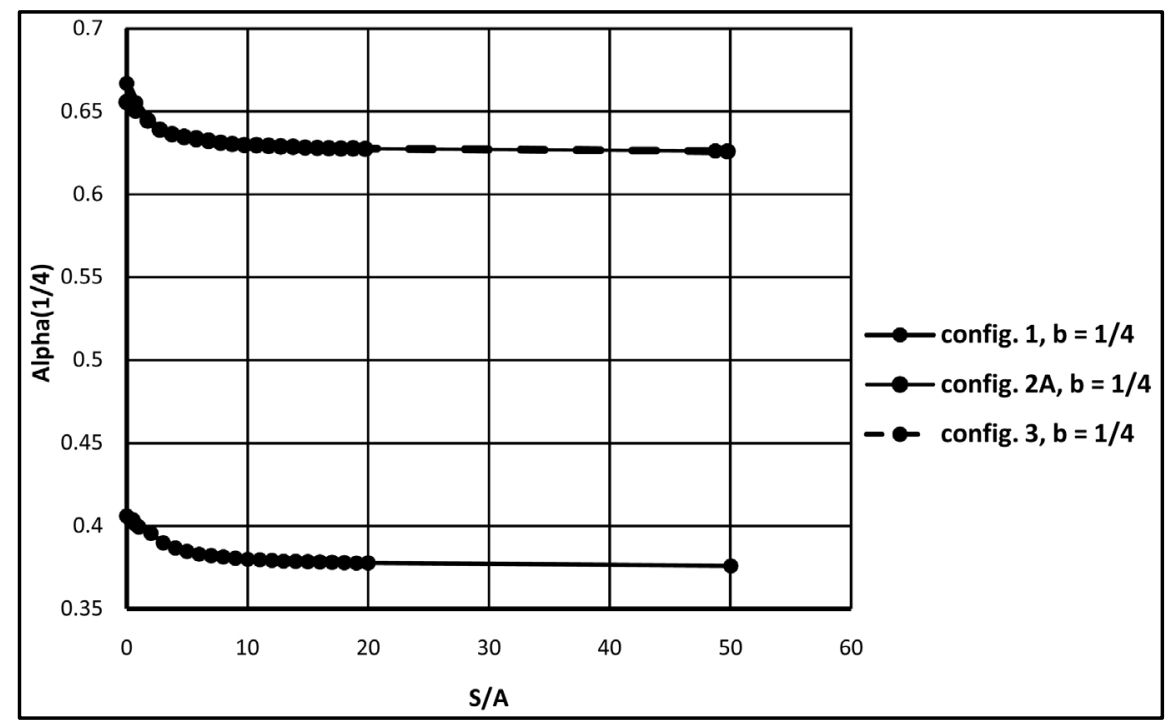

Figure 13. Alpha $1 / 4$ vs. $S / A$ from 0 to 50 A for $b=1 / 4$. The top two curves, which are almost indistinguishable, represent the curved side facing forward, and approach $5 / 8$ at large $S / A$. The bottom curve represents the flat side facing forward and approaches $3 / 8$ at large $S / A$.

the flat or curved side faces the other hemisphere. That condition is denoted by "flat" or "curved" in Figure 12. The curves in Figure 12 and Figure 13 can be interpreted as indicating that the force between the two hemispheres decays away more slowly than $1 / S^{2}$ and only approaches that behavior at very large vales of $S$ (as shown in Figure 10 and Figure 11). As Alpha diminishes, the increasing separation between the two fictitious point masses with $S$ is partially offset by 
their move toward each other within their respective hemisphere.

\subsection{The Use of Power Expansions and Legendre Polynomials}

Without the use of high-speed computers and sophisticated computational software, such as Wolfram Mathematica or, perhaps, MATLAB, it would be extremely difficult to perform the four-fold integration that makes up Equation (5). This is particularly true because of the coupling among the variables of integration. However, the use of a power expansion of the on-axis gravitational potential of hemisphere $A$ (or $B$ ), followed by a term-by-term multiplication by Legendre polynomials to produce off-axis values of the potential simplifies the calculation. This is commonly done when dealing with electrostatics problems containing azimuthal symmetry, and it is discussed in many textbooks [3]. This method can be applied to the current problem because, as in electrostatics, it requires a solution of Laplace's equation. The only possible drawback is that the result is an infinite series, which may require a largenumber of terms to produce the desired accuracy. Fortunately, this will not be true of the number of terms required for an accurate solution to the current problem.

This method of solution for configuration 1 is presented in conjunction with Figure 14, where a hemisphere of radius $A$, assumed to be the source of the potential, $\Phi(R, \theta)$, is shown with the hemisphere of radius $B$, assumed to be in the gravitational field of hemisphere $A$.

Once the potential, $\Phi(r, \theta)$, is determined, its negative $\mathrm{z}$-derivative (the $\mathrm{z}$ component of its gravitational field) is calculated and integrated over hemisphere $B$ to determine the total force between the two hemispheres. In the case of Figure 14, when $R \leq A$, the on-axis potential can be written as a power series in $R^{l}$, whereas for $R \geq A$, it can be written as a power series in $R^{-(l+1)}$. Thus, the potential at angle $\theta$ is of the form:

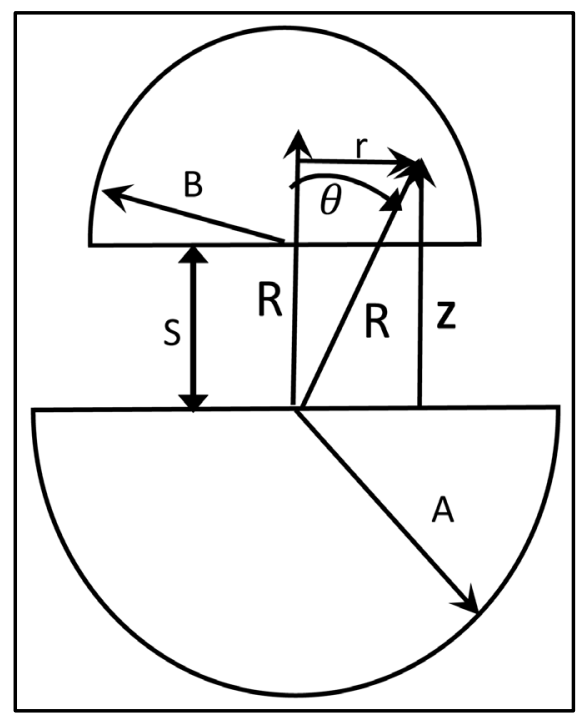

Figure 14. The geometry for the use of power series expansions and Legendre Polynomials for configuration 1. 


$$
\begin{gathered}
\Phi(R, \theta)=\sum_{l=0}^{\infty} C_{l} R^{l} P_{l}(\cos \theta) ; R \leq A \\
\Phi(R, \theta)=\sum_{l=0}^{\infty} D_{l} R^{-(l+1)} P_{l}(\cos \theta) ; R \geq A
\end{gathered}
$$

$C_{l}$ and $D_{l}$ are parameters to be determined and $P_{l}(\cos \theta)$ is the Legendre Polynomial of order 1 , all of which are unity at $\theta=0$. In order to proceed, we must first determine the on-axis potential. After a simple integration it can be shown to be:

$$
\Phi(R, 0)=-G\left(2 \pi \rho_{a}\right)\left[\frac{(A+R)^{3}}{3 R}-\frac{\left(A^{2}+R^{2}\right)^{\frac{3}{2}}}{3 R}-A R-\frac{A^{2}}{2}\right]
$$

Ignoring constants in the above expression, which contribute nothing to the force, results in the following expansions for $R \leq A$ and $R \geq A$, respectively.

$$
\Phi(R, 0)=-G\left(\frac{2 \pi}{3} \rho_{a} A^{3}\right)\left(\frac{1}{A}\right)\left[-\left(\frac{3}{2}\right)\left(\frac{R}{A}\right)+\left(\frac{R}{A}\right)^{2}-\left(\frac{3}{8}\right)\left(\frac{R}{A}\right)^{3}+\left(\frac{1}{16}\right)\left(\frac{R}{A}\right)^{5}-\left(\frac{3}{128}\right)\left(\frac{R}{A}\right)^{7}+\cdots\right]
$$

and

$$
\Phi(R, 0)=-G\left(\frac{2 \pi}{3} \rho_{a} A^{3}\right)\left(\frac{1}{A}\right)\left[\left(\frac{A}{R}\right)+\left(\frac{3}{8}\right)\left(\frac{A}{R}\right)^{2}-\left(\frac{1}{16}\right)\left(\frac{A}{R}\right)^{4}+\left(\frac{3}{128}\right)\left(\frac{A}{R}\right)^{6}-\left(\frac{3}{256}\right)\left(\frac{A}{R}\right)^{8}+\cdots\right]
$$

From these two equations, the constants $C_{l}$ and $D_{l}$ can be directly determined. To obtain $\Phi(R, \theta)$, we multiply each term in the above two expansions by the appropriate Legendre polynomial, express the expansion in terms of the hemispheric coordinates $Z, r, \varphi$ and, to obtain the force, integrate its negative $z$-derivative over $\mathrm{z}$ from $\mathrm{s}$ to $\left(s+\left(B^{2}-r^{2}\right)^{1 / 2}\right)$ and over $\mathrm{r}$ from 0 to $B$. The azimuthal integration over $\varphi$ is simply replaced by $2 \pi$. In addition, we note that $\cos \theta=z /\left(z^{2}+r^{2}\right)^{1 / 2}$. When all of this and a few other manipulations are completed, we identify $\left(1 / R_{\text {eff, } A}\right)^{2}$ for $R \leq A$ as:

$$
\frac{1}{\left(R_{e f f, A}\right)^{2}}=\left(\frac{3}{b^{3}}\right) \int_{0}^{b} r_{a} \mathrm{~d} r_{a} \int_{S_{a}}^{S_{a}+\left(b^{2}-r_{a}^{2}\right)^{1 / 2}} \mathrm{~d} z_{a} \frac{\partial}{\partial z_{a}}\left[\frac{3}{2}\left(z_{a}^{2}+r_{a}^{2}\right)^{\frac{1}{2}}-\left(z_{a}^{2}+r_{a}^{2}\right) P_{2}\left(\frac{z_{a}}{\left(z_{a}^{2}+r_{a}^{2}\right)^{\frac{1}{2}}}\right]+\cdots\right] ; R \leq A
$$

We note the use of scaled coordinates and the fact that the $z_{a}$-integral is particularly simple because it is the integral of a derivative. The integral over $r_{a}$ was done numerically. For $R \geq A$, the results are similar:

$$
\frac{1}{\left(R_{e f f, A}\right)^{2}}=\left(\frac{3}{b^{3}}\right) \int_{0}^{b} r_{a} \mathrm{~d} r_{a} \int_{S_{a}}^{S_{a}+\left(b^{2}-r_{a}^{2}\right)^{1 / 2}} \mathrm{~d} z_{a} \frac{\partial}{\partial z_{a}}\left[-\left(z_{a}^{2}+r_{a}^{2}\right)^{\frac{-1}{2}}-\left(\frac{3}{8}\right)\left(z_{a}^{2}+r_{a}^{2}\right)^{-1} P_{1}\left(\frac{z_{a}}{\left(z_{a}^{2}+r_{a}^{2}\right)^{\frac{1}{2}}}\right)+\cdots\right] ; R \geq A
$$

For simplicity, only the first two terms of each expansion are shown in Equations (10a) and (10b). Figure 15 and Figure 16 show the results of this method for $b=1$ and $b=$ 0.25 , respectively, along with those using Wolfram Mathematica. With only a very few terms of the expansion, the two sets are virtually indistinguishable on the graphs. 


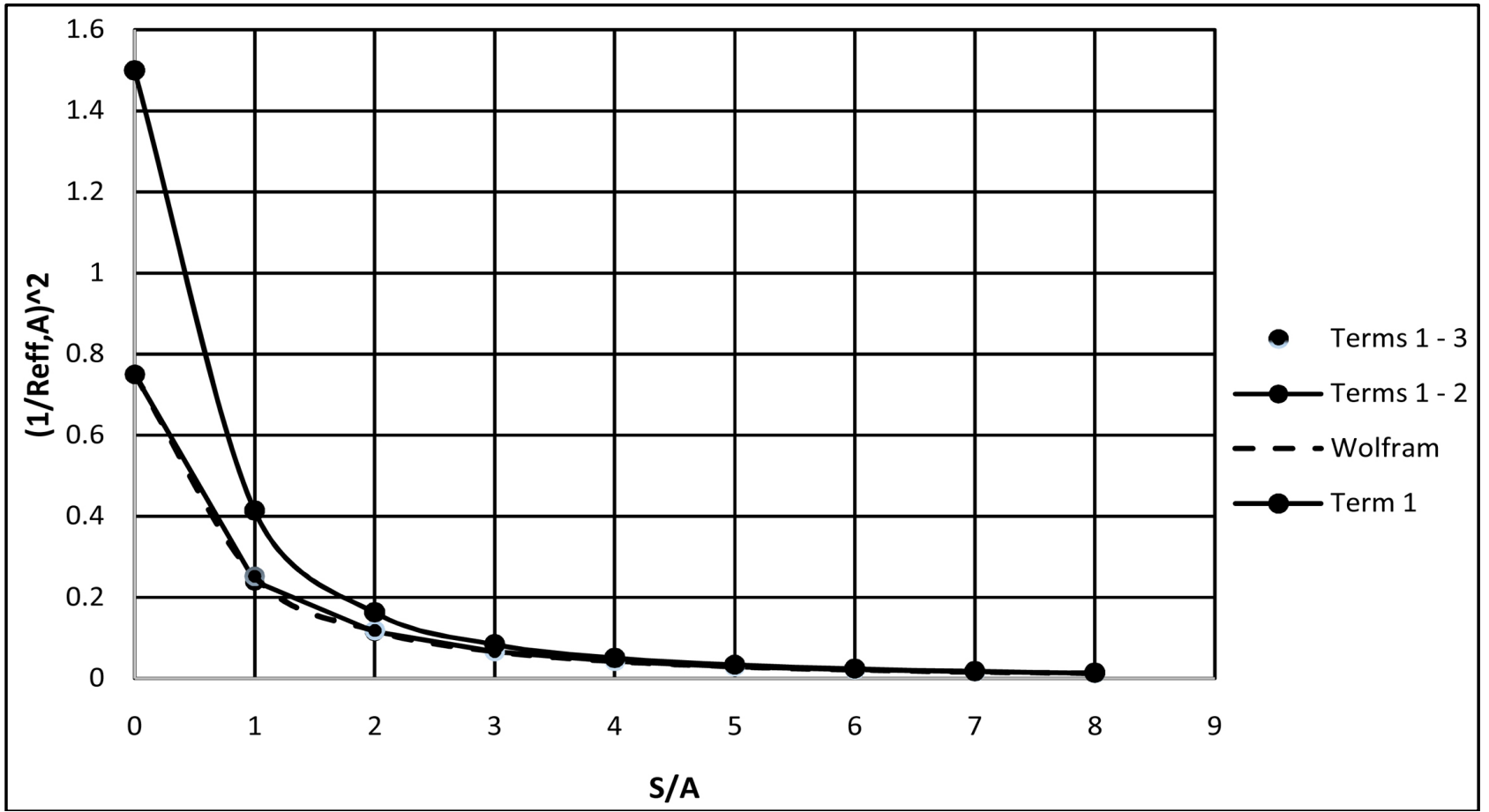

Figure 15. The results of the two methods for $b=1$, showing how well the two methods agree after the use of only a very few terms in the expansion. The results from Wolfram Mathematica, the use of terms $1-2$ of the series, and the use of terms $1-3$ of the series are essentially indistinguishable.

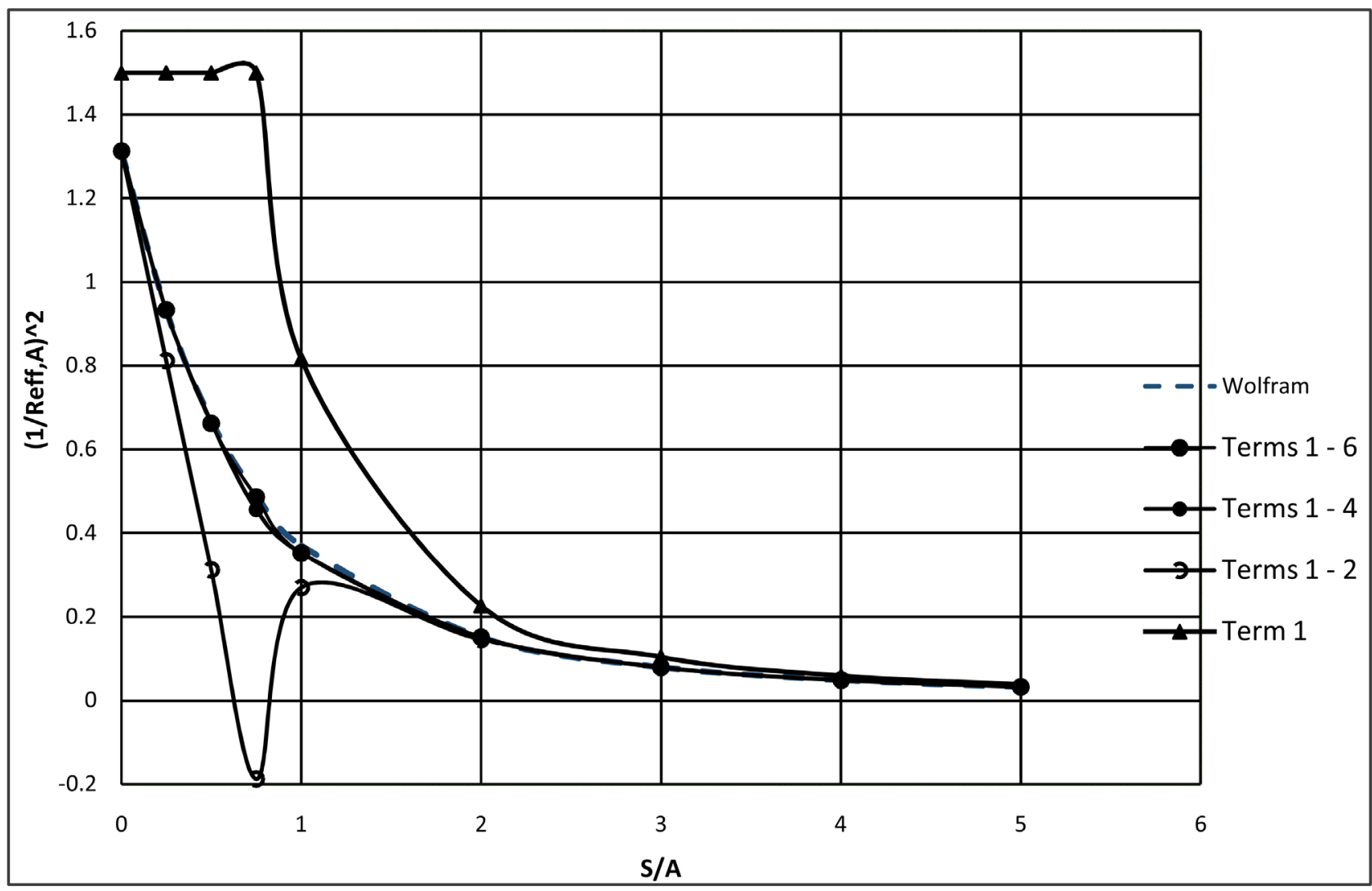

Figure 16. Similar to Figure 15, but for $b=1 / 4$. The results from Wolfram Mathematica, the use of terms $1-4$ of the series, and terms 1 - 6 of the series are essentially indistinguishable. 


\section{Summary}

This paper consists of a study of the gravitational attraction between two inline hemispheres over a range of separations from zero to 50 times the radius of the larger of the two hemispheres. Two relative radii were chosen, 1:1 and 0.25:1 as sufficiently representative, along with three relative orientations: flat side facing flat side, curved side facing curved side, and flat side facing curved side. Initially, the first was calculated through a "brute force", four-fold numerical integration with the help of Wolfram Mathematica. The second and third orientations were derived from the first by a superposition of the first with a simpler, easily calculated, intermediate configuration. The results were expressed in terms of the canonical form of two point masses separated by an effective distance. That "effective distance" was calculated, as was the location of the "point masses" within the hemispheres. An application of the 1:1, flat-to-flat configuration was made to a split, rotating earth. Finally, the flat-to-flat configuration was analyzed using a power series expansion for the on-axis potential and Legendre polynomials. With the use of very few terms in the expansion, the results agreed extremely well with the four-fold integration.

Based on the methods of calculation presented here, future work could involve other pairs of masses that form a cylindrically symmetric configuration. Elongating or compressing one or both hemispheres along their individual axes immediately comes to mind.

\section{Acknowledgements}

The author is grateful to Joseph S. Accetta, CEO of JSA Photonics, and William F. Filter, retired Sandia National Laboratories physicist, for reviewing the manuscript and making valuable suggestions for its improvement.

\section{References}

[1] Gray, A. (1906) Solutions of Physical Problems. Proceedings of the Royal Philosophical Society of Glasgow, 37, 192.

[2] If the earth is cut into two halves, what will be the gravitational force between them? https://www.quora.com/If-the-earth-is-cut-into-two-halves-what-will-be-the-gravit ational-force-between-them

[3] Jackson, J.D. (1999) Classical Electrodynamics. 3rd Edition, John Wiley \& Sons, New York. 
Submit or recommend next manuscript to SCIRP and we will provide best service for you:

Accepting pre-submission inquiries through Email, Facebook, LinkedIn, Twitter, etc. A wide selection of journals (inclusive of 9 subjects, more than 200 journals)

Providing 24-hour high-quality service

User-friendly online submission system

Fair and swift peer-review system

Efficient typesetting and proofreading procedure

Display of the result of downloads and visits, as well as the number of cited articles Maximum dissemination of your research work

Submit your manuscript at: http://papersubmission.scirp.org/

Or contact am@scirp.org 